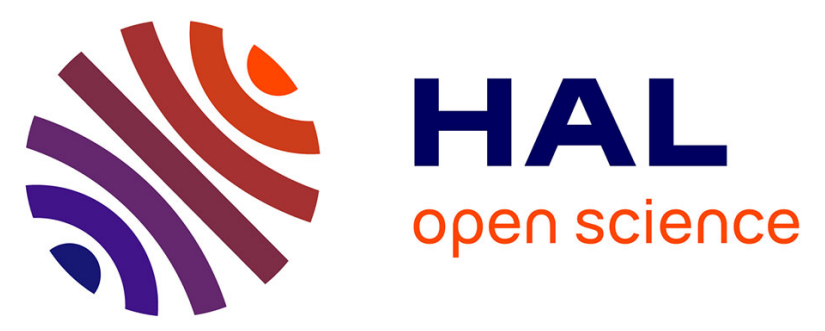

\title{
An experimental analysis of fluid structure interaction on a flexible hydrofoil in various flow regimes including cavitating flow
}

Antoine Ducoin, Jacques Andre Astolfi, Jean-Francois Sigrist

\section{- To cite this version:}

Antoine Ducoin, Jacques Andre Astolfi, Jean-Francois Sigrist. An experimental analysis of fluid structure interaction on a flexible hydrofoil in various flow regimes including cavitating flow. European Journal of Mechanics - B/Fluids, 2012, 36, pp.63-74. 10.1016/j.euromechflu.2012.03.009 . hal-01088167

\section{HAL Id: hal-01088167 \\ https://hal.science/hal-01088167}

Submitted on 13 Jan 2015

HAL is a multi-disciplinary open access archive for the deposit and dissemination of scientific research documents, whether they are published or not. The documents may come from teaching and research institutions in France or abroad, or from public or private research centers.
L'archive ouverte pluridisciplinaire HAL, est destinée au dépôt et à la diffusion de documents scientifiques de niveau recherche, publiés ou non, émanant des établissements d'enseignement et de recherche français ou étrangers, des laboratoires publics ou privés. 


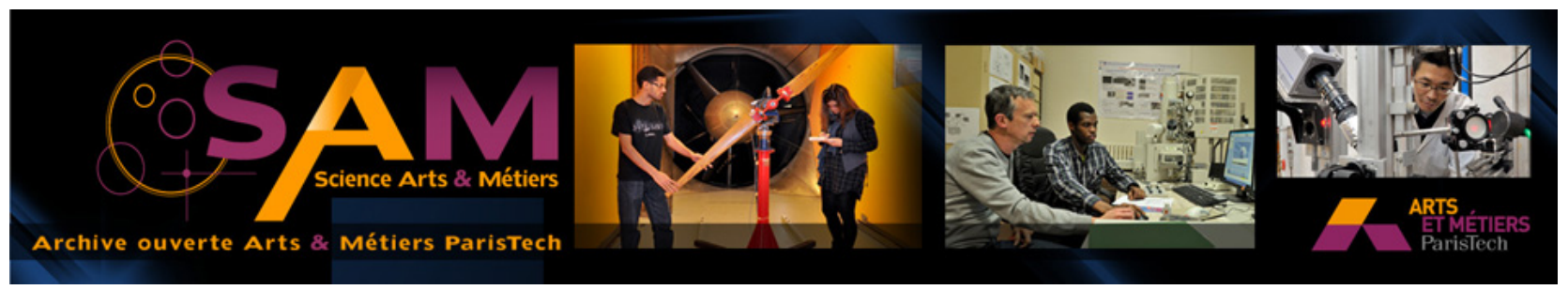

Science Arts \& Métiers (SAM)

is an open access repository that collects the work of Arts et Métiers ParisTech researchers and makes it freely available over the web where possible.

This is an author-deposited version published in: http://sam.ensam.eu

Handle ID: .http://hdl.handle.net/10985/8998

\section{To cite this version :}

Antoine DUCOIN, Jacques Andre ASTOLFI, Jean-François SIGRIST - An experimental analysis of fluid structure interaction on a flexible hydrofoil in various flow regimes including cavitating flow - European Journal of Mechanics - B/Fluids - Vol. 36, p..63-74. - 2012 


\title{
An experimental analysis of fluid structure interaction on a flexible hydrofoil in various flow regimes including cavitating flow
}

\author{
Antoine Ducoin ${ }^{\mathrm{a}, *}$, Jacques André Astolfi ${ }^{\mathrm{b}}$, Jean-François Sigrist ${ }^{\mathrm{c}}$ \\ a University of Michigan, Department of NAME, 2600 Draper Dr, 48109 Ann Arbor, MI, USA \\ ${ }^{\mathrm{b}}$ Institut de Recherche de l'Ecole Navale EA 3634, BRCM Brest, Ecole Navale C600, 29240 Brest cedex 9, France \\ ${ }^{\mathrm{c}}$ DCNS Propulsion, Nantes, France
}

\section{Keywords:}

Hydroelasticity

Lifting bodies

Cavitation

Transient regimes

\begin{abstract}
A B S T R A C T
The structural response of a rectangular cantilevered flexible hydrofoil submitted to various flow regimes is analyzed through an original experiment carried out in a hydrodynamic tunnel at a Reynolds number of $0.75 \times 10^{6}$. The experiment considers static and transient regimes. The latter consists of transient pitching motions at low and fast pitching velocities. The experiments are also performed for cavitating flow. The structural response is analyzed through the measurement of the free foil tip section displacement using a high speed video camera and surface velocity vibrations using a laser doppler vibrometer.

In non cavitating flows, it is shown that the structural response is linked to the hydrodynamic loading, which is governed by viscous effects such as laminar to turbulent transition induced by Laminar Separation Bubble (LSB), and stall. It is also observed that the foil elastic displacement depends strongly on the pitching velocity. Large overshoots and hysteresis effect are observed as the pitching velocity increases. Cavitation induces a large increase of the vibration level due to hydrodynamic loading unsteadiness and change of modal response for specific frequencies. The experimental results presented in this paper will help to develop high fidelity fluid-structure interaction models in naval applications.
\end{abstract}

\section{Introduction}

The knowledge of the hydroelastic behavior of flexible lifting bodies is fundamental for the design and sizing of recent naval systems such as the new concepts of propulsion systems based on composite marine propellers [1], active control surfaces like rudders [2] or innovative tidal turbine blades [3,4]. For a flexible body working in a steady flow, an equilibrium state is obtained between the deformed shape and the steady hydrodynamic loading [5]. However under specific or extreme operating conditions, a sudden change of incidence or inflow velocity can lead to transient regimes that may induce strong unsteady hydrodynamic loadings. This is often coupled with boundary layer unsteady characteristics like partial or large boundary layer detachment, sudden laminar to turbulent transition or unsteady cavitation. In that case, the high amplitude fluctuations of the hydrodynamic loading interacting with a flexible body can induce strong fluid structure interactions and possible occurrence of hydro-elastic instabilities. Several experiments have been performed to study the flow over pitching foils. The major part of the studies are related to oscillating rigid airfoils.

\footnotetext{
* Corresponding author. Tel.: +1 7348349195.

E-mail address: antoine.ducoin@laposte.net (A. Ducoin).
}

Particularly, the knowledge of the wall pressure field distribution and time evolution on the body brought new elements in the understanding of dynamic loading induced by pitching motions [6,7]. Jumper et al. [6] performed wall pressure measurements on a rigid hydrofoil for different pitching velocities $\dot{\alpha}$ and upstream velocities $V_{\infty}$. They showed that the unsteady nature of the hydrodynamic loading is related to the similarity parameter $\dot{\alpha}^{*}=\dot{\alpha} c / V_{\infty}$, where $c$ is the chord length. This parameter gives the foil rotation, during the time of a particle that moves from the leading edge to the trailing edge. Moreover, the stall was clearly delayed when $\dot{\alpha}$ increases. It was shown that the unsteady nature of the flow influences the hydrodynamic loading [8]. Lee and Gerontakos [9] focused on the effect of the laminar to turbulent transition downstream a Laminar Separation Bubble (LSB) for an oscillating airfoil at various pitching velocities at $\operatorname{Re}=1.35 \times 10^{5}$. For small values of the pitching velocity, they showed that the LSB induced variations in lift, drag and moment coefficients. As well, boundary layer transition was delayed and promoted when the pitching velocity increased. This conducted to the modification of lift coefficients and lift-curve slope. However, it was shown that the Laminar Separation Bubble characteristics are insensitive to pitching velocity. Similar conclusions are drawn for a transient pitching hydrofoil [10].

On the other hand, cavitation is of primary importance for marine structure design [11]. Depending on the flow conditions, different kind of cavitation can occur [12]. It is known that under 


\begin{tabular}{|ll}
\hline \multicolumn{2}{l}{ Nomenclature } \\
$b$ & Hydrofoil span \\
$c$ & Hydrofoil chord \\
$C_{L}^{+}$ & Suction side lift coefficient \\
$E$ & Young's Modulus \\
$h$ & Tunnel test section height \\
$P_{0}$ & Reference pressure \\
$P_{V}$ & Vapor pressure \\
$\operatorname{Re}$ & Reynolds number $\left(=V_{\infty} c / v\right)$ \\
$V_{\infty}$ & Free stream velocity
\end{tabular}

\section{Greek letters}

$\alpha \quad$ Angle of incidence

$\alpha^{+} \quad$ Angle of incidence, upward pitching motion

$\alpha^{-} \quad$ Angle of incidence, downward pitching motion

$\dot{\alpha} \quad$ Pitching velocity

$\dot{\alpha}^{*} \quad$ Dimensionless pitching velocity $\left(=\dot{\alpha} c / V_{\infty}\right)$

$\delta_{0} \quad$ Rigid structure displacement

$\delta_{t} \quad$ Total structure displacement

$\delta_{y} \quad$ Elastic structure displacement

$\rho_{F} \quad$ Fluid density

$\rho_{F} \quad$ Solid density

$v_{F} \quad$ Fluid kinematic viscosity

$v_{S} \quad$ Poisson ratio

unfavorable conditions, the vaporized area becomes unstable, with cavity break-off and periodical shedding of large bubble clusters. This configuration, usually called "cloud cavitation", generates pressure fluctuations downstream of the cavity [13] and could have strong impacts when developing on flexible structures.

Cavitation have also been considered for pitching hydrofoils. In [14], numerical results of a pitching Joukowski hydrofoil with sheet-cloud are compared to experiments. The numerical method is based on potential flow. For low reduced frequency $k$, large cavity oscillations are observed whereas higher reduced frequency leads to a progressive cavity response and a rapid collapse. In that case, $k$ appears to be close to the cavity natural shedding frequency $\omega_{n}$. In [15], the effect of pitching velocity on cavitation was observed experimentally on a NACA64 hydrofoil. A phase lag was found between the extending of the cavity and the oscillation that increases with $k$. A strong interaction between $k$ and $\omega_{n}$ was observed and the cavitation behavior becomes random when $k \approx$ $\omega_{n}$. For the highest $k$, a loud pounding sound resulting from the collapse of the cavity and a strong hysteresis between cavitation inception and desinence was observed.

Recent efforts were made in order to approach through simulations both the fluid and the structure dynamics. This comes from the recent development of numerical methods and models which allow us to compute complex flows together with coupled simulation with reasonable CPU time [16]. For aerodynamic applications, studies have highlighted that structural deformation under static or dynamic loads may be of primary importance in the evaluation of aerodynamic performances [17]. Bhardwaj [18] developed a CSD/CFD coupling procedure where the importance of the computation initialization from a CFD steady-state solution was emphasized. Send [19] and Patil and Hodges [20] investigated the correlation between the wing deformation (mainly twist) and variation of the aerodynamic coefficients such as lift and wall pressure coefficients. On the other hand, few works show concern for hydro-elasticity applications. In [21] developed a coupled procedure solved in the frequency domain for blade stress analysis on propellers. Non linear hydroelastic behavior of propellers has also been investigated by Lin and Lin [22] using lifting surface theory. Amromin and Kovinskaya [23] analyzed theoretically the vibration of an elastic wing with an attached cavity in a periodically perturbed flow. Wing vibration was described by means of a simple beam equation. As a result, two frequency bands were found. A high-frequency band associated mainly with the elastic resonant natural frequencies of the foil and a low-frequency band associated with cavity-volume oscillations. Ausoni et al. [24] have studied experimentally the interaction between cavitating Von Karman vortices in the wake of a NACA009 hydrofoil and its vibrations. In lock in conditions (resonance), the cavitation inception is enhanced and in fully developed cavitating condition, the vibrations of the first tortionnal mode increase the strouhal cavity shedding frequency. Reese [25] have examined vibrations and damping of a NACA66 hydrofoil in uniform flow. He showed that the structural damping decreases when the vortex frequency gets close to a natural frequency, whereas the added stiffness and hydrodynamic damping becomes significant at high speeds. Recently, Young [16] presented a new approach applied to a cavitating propeller which takes into account the modification of fluid pressure field induced by the blade displacement. In [26,1, 27], the fluid structure interaction is used to increase the efficiency of marine propellers. Composite material is used to control the deformation of the blade in order to reduce the cavitation and to increase the efficiency. This has been recently applied for the design and optimization of adaptive marine propellers [28] and tidal turbines blades [3].

The numerical works are very interesting and bring new results, however it appears that there are very few experimental data in fluid structure interaction applied to lifting bodies in hydrodynamic applications. The aim of the paper is to get a better understanding of fluid structure interaction in hydrodynamic applications and to provide experimental data for the development of high fidelity fluid structure interaction models. A previous study concerning the investigation of the flow over a flexible hydrofoil in uniform flow was performed (see [5]). The present paper gives extended experimental results by including stationnary hydrofoil and transient pitching motions. The system was modified in order to obtain transient pitching motion at low, medium and high pitching velocities and to measure the structural dynamics. Cavitation-Structure Interaction is also shown, for both stationary and pitching hydrofoils. The vibrations and displacements of the tip are measured together with the cavitation observed at the suction side of the hydrofoil. The comparison with subcavitating flow highlights the possible effects of cavitation on hydroelastic response.

\section{Experimental setup}

Measurements were carried out at the cavitation tunnel of the Research Institute of the French Naval Academy. The test section is $0.192 \mathrm{~m}^{2}$ and $1 \mathrm{~m}$ long. The velocity ranges between 3 and $15 \mathrm{~m} / \mathrm{s}$ and the pressure in the test section ranges between 30 mbar and 3 bar. A flexible hydrofoil is mounted horizontally at mid height of the test section, see Fig. 1. It is clamped at the root section and free at the tip.

The hydrofoil has an NACA66312 section. It has a camber type line $a=0.8$, a camber ratio of $2 \%$ and a relative thickness of $12 \%$ [13]. The chord is $c=0.148 \mathrm{~m}$ and the span is $b=0.191 \mathrm{~m}$. It corresponds to an aspect ratio $b / c=1.3$ and a confinement parameter $h / c=1.28$. These relatively small ratios can induce $3 \mathrm{D}$ effects, induced by the development of boundary layers of the vertical walls that interact with the foil surface in the vicinity of the tip and the root of the hydrofoil. It was observed that the wall boundary layer thickness was of about $0.009 \mathrm{~m}$ for a free stream velocity of $5 \mathrm{~m} / \mathrm{s}$ corresponding to about $10 \%$ of the span. However flow visualizations based on cavitation inception and cavity development showed that the flow can be considered 


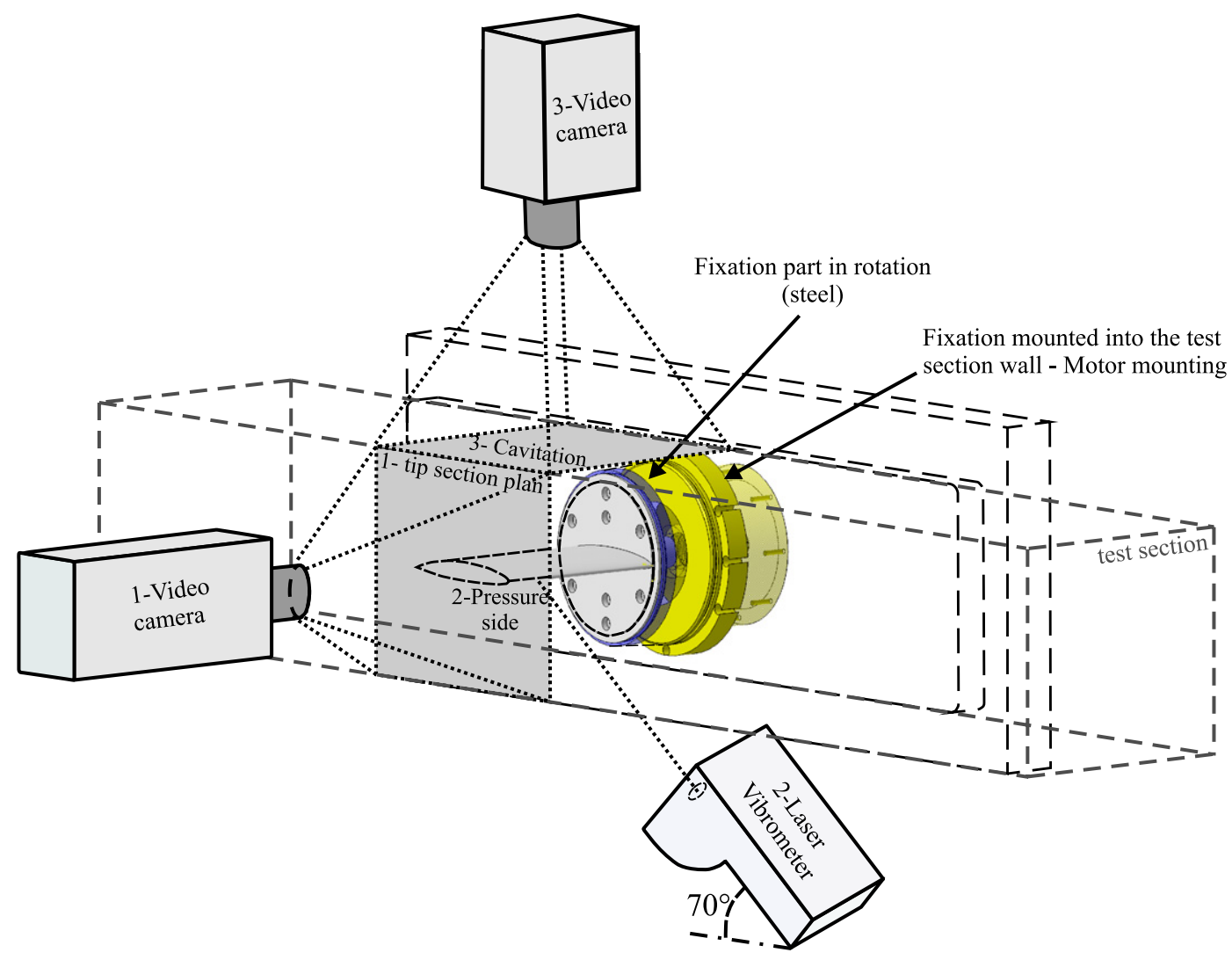

Fig. 1. Experimental set up.

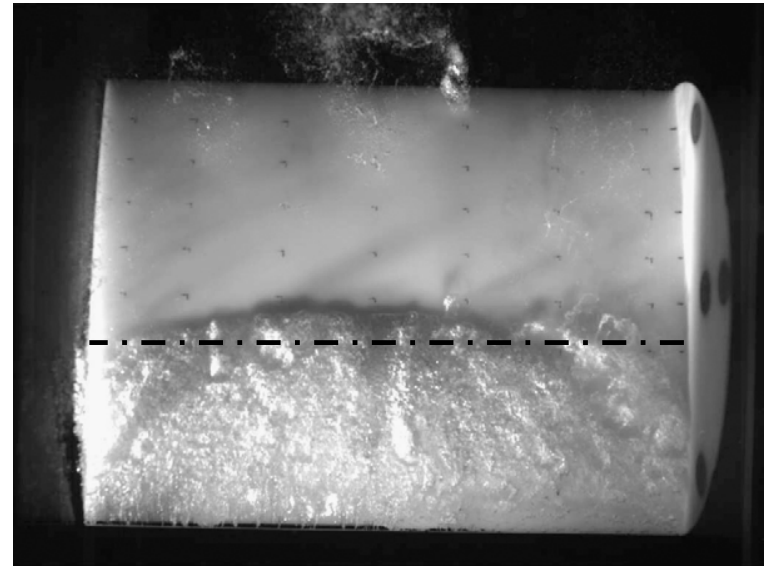

Fig. 2. Typical cavitation pattern obtained from the high speed camera (top view), $\alpha=8^{\circ}$.

reasonably bidimensional on a major part of the foil span as shown in Fig. 2 (see also [29]). The gap between the hydrofoil tip and the vertical wall is about $1 \mathrm{~mm}$, which is lower that the wall boundary layer thickness. As shown later in the paper, this can however induce development of tip vortex and tip leakage cavitation, which is seen to have negligible effects on the hydroelastic response.

The flexible hydrofoil has a Polyacetate (POM) material $(E=$ $\left.3000 \mathrm{MPa}, \rho_{S}=1480 \mathrm{~kg} / \mathrm{m}^{-3}, v_{S}=0.35\right)$. The system is composed of the hydrofoil and a disc fabricated in one-piece to ensure a clamped condition at the root section. The disc is fixed with eight screws to a steel disc. The system is finally fixed to a cylinder that is linked to the motor housing to ensure the foil rotation. A video camera BASLER at a sampling frequency of 100 images per second is used in order to get a side view of the hydrofoil and to measure the instantaneous displacement of the tip section (see video camera 1 in Fig. 1).

The total displacement $\vec{\delta}_{t}^{M}(t)$ of a point $M(x, y)$ about the origin $O\left(x_{0}, y_{0}\right)$ (center of rotation) of the foil tip section can be split in two parts:

$\vec{\delta}_{t}^{M}(t)=\vec{\delta}_{o}^{M}(t)+\vec{\delta}^{M}(t)$

where $\vec{\delta}_{o}^{M}(t)$ is the rigid solid displacement of an arbitrary point $M$ at the hydrofoil tip due to the rigid foil rotation and $\vec{\delta}^{M}(t)$ is the elastic displacement of the same arbitrary point due to the foil deformations induced by the hydrodynamic loading.

From Eq. (1) we have:

$\vec{\delta}^{M}(t)=\vec{\delta}_{t}^{M}(t)-\vec{\delta}_{o}^{M}(t)$

Without flow, no foil elastic deformation occurs; the displacement is only due to solid rotation. Then, $\vec{\delta}_{o}^{M}(t)$ and $\vec{\delta}_{t}^{M}(t)$ can be easily determined experimentally for a given pitching velocity. The pictures with and without flow velocity are processed to calculate the displacements, as shown in Fig. 3. Moreover, in the plane of the foil tip section, the elastic displacement has two components:

$\vec{\delta}^{M}(t)=\delta_{x}^{M}(t) \vec{i}+\delta_{y}^{M}(t) \vec{j}$.

It was observed that the elastic displacement is mainly due to the vertical displacement (i.e. bending), then we have:

$\vec{\delta}^{M}(t) \approx \delta_{y}^{M}(t) \vec{j}$.

For simplicity, the vertical elastic displacement will be noted $\delta_{y}(t)$.

Reading uncertainty is about \pm 1 pixel. Since 1 pixel equals $0.07 \mathrm{~mm}$, the uncertainty on measured displacements is $\Delta(y)=$ $\pm 0.07 \mathrm{~mm}$. The corresponding non dimensional uncertainty is $\Delta(y) / b= \pm 0.00037$, where $b$ is the span of the hydrofoil equal to $191 \mathrm{~mm}$. 


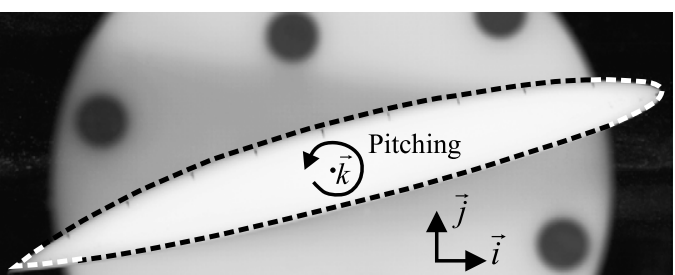

(a) $V_{\infty}=0 \mathrm{~m} / \mathrm{s}$.

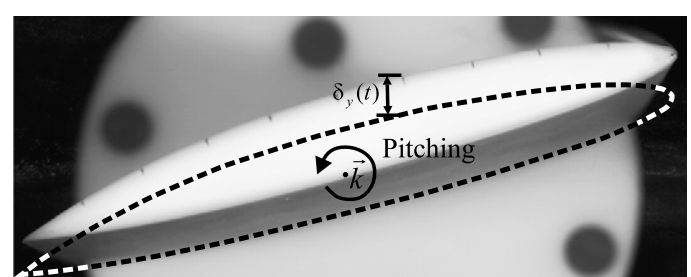

(b) $V_{\infty}=7 \mathrm{~m} / \mathrm{s}$.

Fig. 3. Measurement of the free tip section.

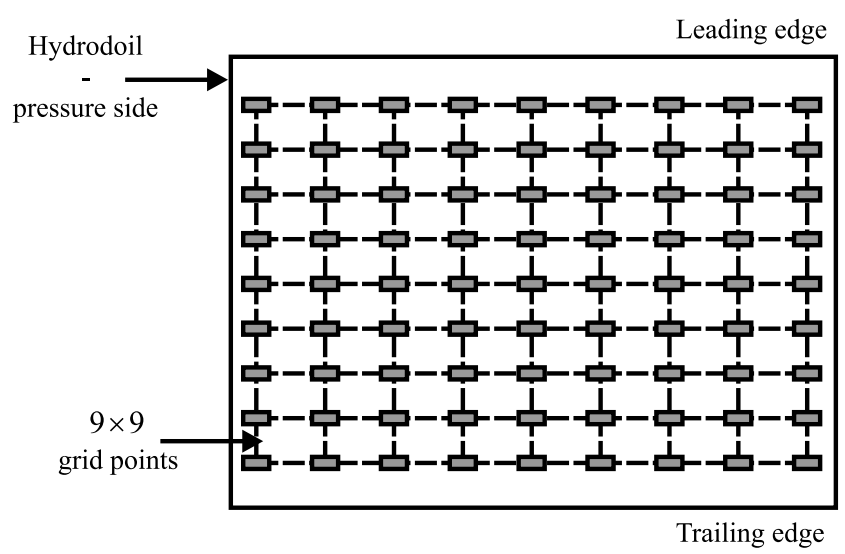

Fig. 4. Mesh grid on the foil surface for the vibration measurements.

The analysis of structural vibrations is carried out on the hydrofoil pressure side using a Laser Doppler Vibrometer Polytech PSV-400 (Fig. 1). It is a class II He-Ne laser of wavelength $\lambda=$ $632.8 \mathrm{~nm}$. The vibrometer sensitivity ranges between $10 \mathrm{~mm} / \mathrm{s} / \mathrm{V}$ and $1 \mathrm{~m} / \mathrm{s} / \mathrm{V}$. The system performs scanning measurements over a grid pre-defined on the structure surface (Fig. 4) by means of an internal camera. A mean vibration spectrum was obtained from an average of 32 spectrums obtained on one point of the grid. Then the spectrum could be averaged over the surface to obtain the average spatial vibration spectrum of the surface.

Finally, the cavitation is observed at the suction side with a high speed camera PHOTRON on the top of the test section (Fig. 1) at a sampling frequency of 2000 images per second. The cavitation condition is based on the cavitation number defined as $\sigma=$ $\left(P_{0}-P_{V}\right) /\left(1 / 2 \rho_{F} V_{\infty}^{2}\right)$ where $P_{0}$ is the pressure in the test section (measured at about two chords upstream of the hydrofoil) and $P_{V}$ is the vapor pressure at ambient temperature. The cavitation was controlled by decreasing $P_{0}$ progressively in the tunnel test section.

The transient pitching motion was defined as a single upward-downward motion from $\alpha=0^{\circ}$ to the maximum incidence $\alpha_{\max }$. The rotation axis is located at $x / c=0.5$ from the leading edge. The mean angular velocity is $\dot{\alpha}=2 \alpha_{\max } / t_{f}$, where $t_{f}$ is the final time of the transient motion. Four pitching velocities were considered, from a slow mean angular velocity of $6 \%$ s (quasi-static) to a high mean angular velocity of $63 \%$ s. The pitching laws giving the angle of incidence versus time are shown on Fig. 5. Two phases of acceleration and deceleration are observed for the highest pitching velocity (Fig. 5(d)). The non-dimensional angular velocity based on the chord length $c$ and the upstream velocity $V_{\infty}$ can be introduced as $\dot{\alpha}^{*}=\dot{\alpha} \times c / V_{\infty}$. The values of $\dot{\alpha}^{*}$ are also given on Fig. 5 for $V_{\infty}=5 \mathrm{~m} / \mathrm{s}$. The maximum angle of $\alpha_{\max }$ was set to $15^{\circ}$ in the present measurements, which is just beyond the angle of static stall close to $13^{\circ}$. The major uncertainty on the foil angular position comes from the initial zero angle of incidence, corresponding to the chord line parallel to the upstream velocity $V_{\infty}$. It was first adjusted by aligning the foil chord line to the horizontal top wall of the test section using a micrometer. Then the chord line was compared to the horizontal free surface line when the tunnel test section was half fulfilled in the vicinity of the foil pressure side. The comparison was done by image processing of lateral pictures. If necessary, the zero angle of incidence was adjusted iteratively in such a way that the horizontal free surface line was parallel to the chord line on the picture. By several trials, with this method, the uncertainty on the angle of incidence was estimated to be $\Delta \alpha= \pm 0.15^{\circ}$.

The nominal free stream velocity $V_{\infty}$ is $5 \mathrm{~m} / \mathrm{s}$, corresponding to a Reynolds number based on the foil chord length of $\operatorname{Re}=750000$. Uncertainties for the free stream velocity is $\Delta V_{\infty}= \pm 0.02 \mathrm{~m} / \mathrm{s}$. The pressure in the tunnel test section was set to $P_{0}=1.4$ bar with an uncertainty of $\Delta P_{0}= \pm 0.003$ bar. Uncertainty of $\sigma$ is about \pm 0.02 .

\section{Results and discussions}

\subsection{Non cavitating flow}

\section{Static case}

It was shown in [10] that the flow experienced different regimes when the angle of incidence is modified, leading to a relative complex flow. For low angles of incidences $\left(\alpha<5^{\circ}\right)$, the boundary layer was laminar over the hydrofoil surface up to $x / c=0.7-0.8$ for which an LSB took place. For $\alpha>5^{\circ}$, the LSB moved suddenly toward the leading edge and the boundary layer was turbulent over the major part of the hydrofoil surface. Moreover, a turbulent boundary layer detachment was formed at the trailing edge and developed as the angle of incidence increase. The static displacement of the tip section is shown in Fig. 6 for different angles of incidences before stall. As shown, the displacement increases linearly up to $5^{\circ}$, then the plateau behavior is observed up to $6.5^{\circ}$ due to the laminar to turbulent transition. For higher angles of incidences, the displacements are affected by the trailing edge boundary layer detachment.

Fig. 7 presents the mean spatial velocity vibration spectrum for three angles of incidences. Peaks corresponding to the natural frequencies of the hydrofoil can be observed: $f_{1}=43 \mathrm{~Hz}$ corresponds to the first bending mode, $f_{2}=171 \mathrm{~Hz}$ is the first torsional mode, $f_{3}=291 \mathrm{~Hz}$ is the second bending mode and $f_{4}=560 \mathrm{~Hz}$ is the first mixed bending and torsional mode. For $\alpha=4^{\circ}$, a frequency of $510 \mathrm{~Hz}$ is observed. It is linked to the LSB induced laminar to turbulent transition at the trailing of the hydrofoil, which generate a high level of fluctuations close to the natural frequency at $f_{4}=560 \mathrm{~Hz}$. For more details about the laminar to turbulent transition characteristics and induced vibrations, see [30]. At $\alpha=8^{\circ}$, no shedding process was observed in the flow and the amplitudes peaks are low, resulting from a low level of periodic fluctuations around the body. When stall occurs at $\alpha=14^{\circ}$, the low frequency of the leading edge vortex shedding highly excite the first bending mode. The frequency peaks of modes 7 and 8 are also observed which are excited by smaller structures of the leading edge vortex shedding. A peak around $f=1900 \mathrm{~Hz}$ were observed on wallpressure fluctuations. The level of vibration is relatively high for low angles of incidences resulting from the LSB fluctuations. It decreases suddenly the transition move to the leading edge. The highest fluctuation level is observed when stall occurs. 


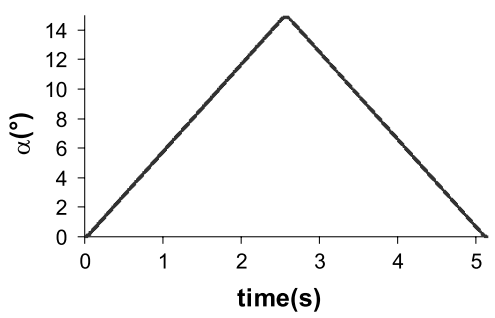

(a) $\dot{\alpha}=6 \%$ s, $\dot{\alpha}^{*}=0.18$.

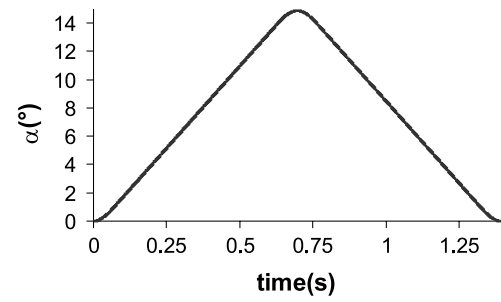

(b) $\dot{\alpha}=20.6^{\circ} / \mathrm{s}, \dot{\alpha}^{*}=0.618$.

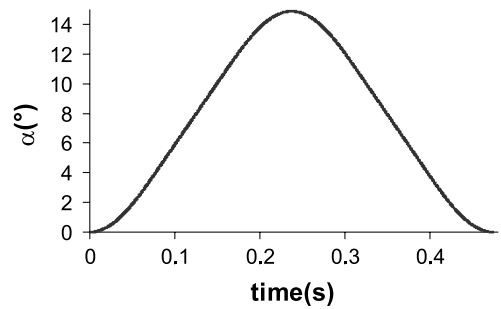

(d) $\dot{\alpha}=63^{\circ} / \mathrm{s}, \dot{\alpha}^{*}=1.89$

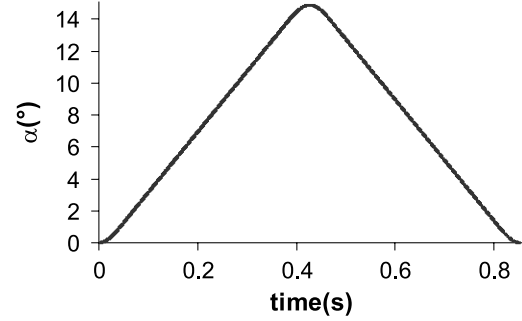

(c) $\dot{\alpha}=35^{\circ} / \mathrm{s}, \dot{\alpha}^{*}=1.05$.

Fig. 5. Pitching velocities, angle of incidence versus time, $V_{\infty}=5 \mathrm{~m} / \mathrm{s}$.

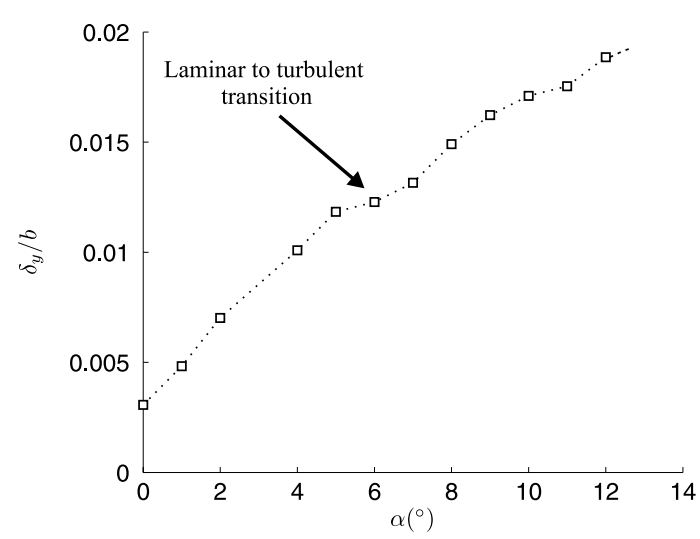

Fig. 6. Static displacement for various angles of incidence, $V_{\infty}=5 \mathrm{~m} / \mathrm{s}$.

\section{Dynamic case}

The tip section displacement was measured for the four pitching velocities. An example of a typical visualization is shown in Fig. 8 at a maximal angle of incidence equal to $15^{\circ}$. The non deformed tip section (zero flow velocity) is given by the dashed line on the picture. The hydrofoil displacement is clearly observable when comparing the non deformed tip section to the deformed one. It was observed that the elastic displacement is mainly vertical (flexion) and that twist is small. When comparing the quasi-static case on Fig. 8(a) to the faster angular velocity on Fig. 8(b), it is observed that the displacement increases as the pitching velocity increases.

The dynamic vertical displacement is shown on Fig. 9 for $\alpha=0^{\circ}$ to $\alpha=15^{\circ}$ with an angular step of $\Delta \alpha=0.5^{\circ}$ for the four pitching velocities during the upward and downward motion. For clarity, the angle of incidence during upward motion (respectively downward) will be denoted $\alpha^{+}$(respectively $\alpha^{-}$). It is reminded that the angle of incidence is deduced from the pitching laws giving the angle of incidence versus time (see Fig. 5). Also plotted on Fig. 9 is the static displacement and the experimental suction side pressure loading $\left(C_{L}^{+}\right)$on a rigid hydrofoil measured by means of a wall-pressure array for the same flow conditions. It is obtained by summing the instantaneous pressure coefficients of the wallpressure array over the suction side [10].

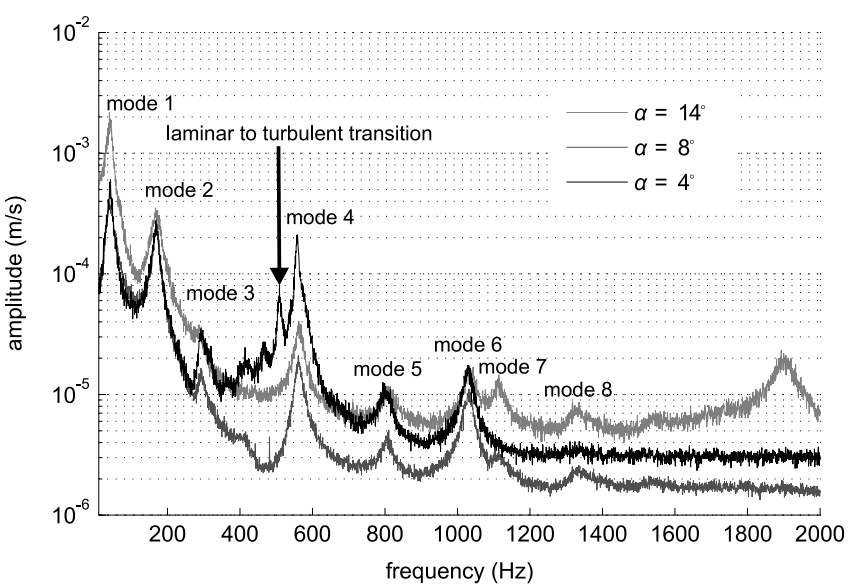

Fig. 7. Velocity spectrum for various angles of incidence, static case, $\mathrm{Re}=$ $750000, V_{\infty}=5 \mathrm{~m} / \mathrm{s}$

From $\alpha^{+}=0^{\circ}$ to $5^{\circ}$ (upward motion), the hydrofoil displacement increases linearly with the instantaneous hydrodynamic loading. When $\alpha^{+}$is close to $5^{\circ}$, an inflection is observed and the displacement is relatively constant and slightly reduced (Fig. 9(a)). It was shown that this is the consequence of the leading edge laminar to turbulent transition. As the pitching velocity increases, the inflection point is delayed and its intensity reduced, it is suppressed for the highest pitching velocity (Fig. 9(d)). This agrees with the flow loading that exhibits similar trends. Beyond the inflection point the displacement increases again together with the loading. It is higher as the pitching velocity increases. At $13^{\circ}$ it is $\delta_{y} / b=0.017$ for $\dot{\alpha}^{*}=0.18$ and $\delta_{y} / b=0.023$ for $\dot{\alpha}^{*}=1.89$. Beyond $13^{\circ}$ (static stall angle), strong displacement fluctuations are observed. Three peaks are observed for $\dot{\alpha}^{*}=0.18$ : a high amplitude peak at $\alpha^{+}=14.5^{\circ}$ followed by two lower peaks at respectively $\alpha^{-}=14.5^{\circ}$ and $\alpha^{-}=13^{\circ}$ (during the downward motion). This agrees with the flow loading exhibiting high amplitude fluctuations resulting from vortex shedding and stall. For the highest pitching velocity, one peak is observed. During the backward motion, from $\alpha^{-}=13^{\circ}$ up to $0^{\circ}$, an hysteresis effect is observed. It is amplified when the pitching velocity increases.

The effect of upstream flow velocity is also be investigated by comparing two measurements with the same pitching velocity and 


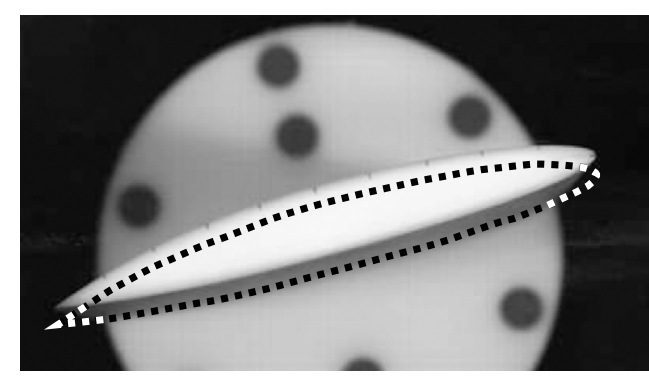

(a) $\dot{\alpha}^{*}=0.12$.

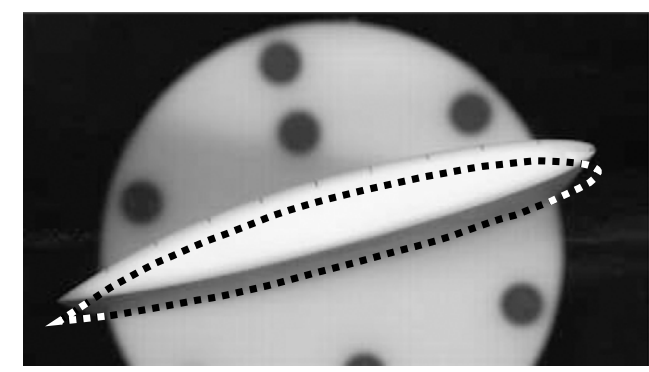

(c) $\dot{\alpha}^{*}=0.75$.

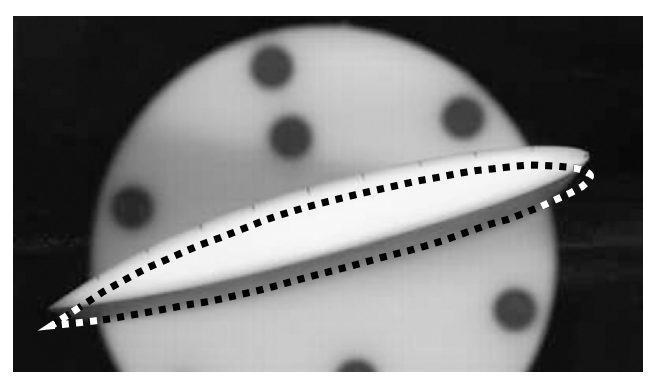

(b) $\dot{\alpha}^{*}=0.44$.

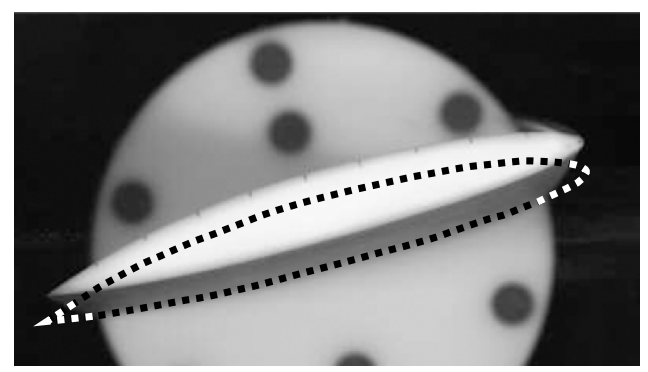

(d) $\dot{\alpha}^{*}=1.35$

Fig. 8. Tip section measurement for 4 pitching velocities at $\alpha^{+,-}=15^{\circ}, V_{\infty}=7 \mathrm{~m} / \mathrm{s}$.
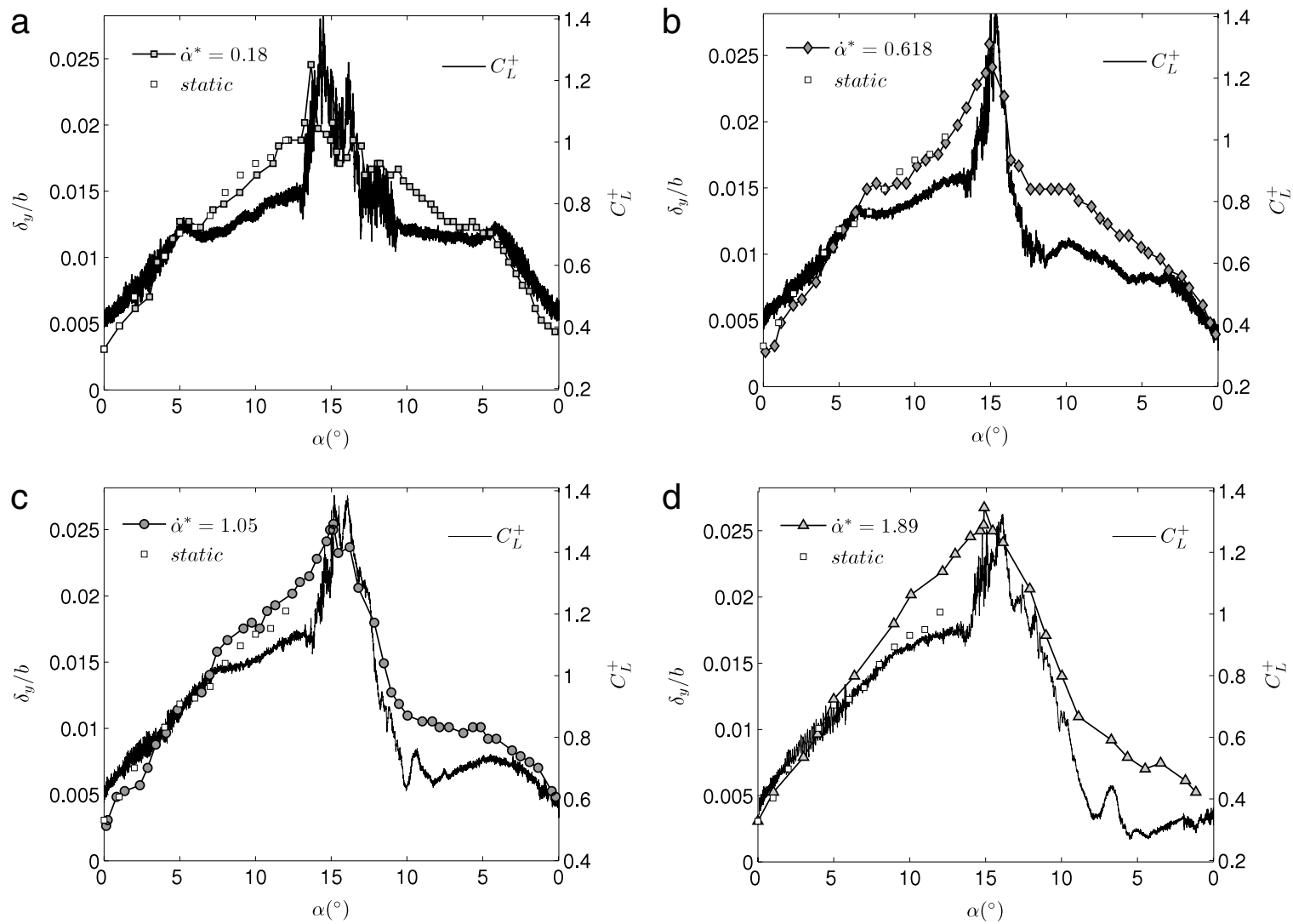

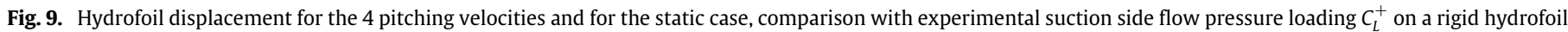
measured by means of a wall-pressure array for the same flow conditions, $\operatorname{Re}=750000, V_{\infty}=5 \mathrm{~m} / \mathrm{s}$.

two different flow velocities. The displacements are divided by the square of the upstream velocity, on which they are proportional to, if the viscous effects are neglected (i.e. potential flow). Fig. 10 shows the results obtained for $\alpha=6 \%$, $V_{\infty}=5 \mathrm{~m} / \mathrm{s}$ and $7 \mathrm{~m} / \mathrm{s}$, leading to respectively $\dot{\alpha}^{*}=0.18$ and $\dot{\alpha}^{*}=0.12$, which are both quasi-static cases. As shown, the displacement level is quite the same when divided by the square of the velocity. It is observed that the effect of transition on displacement $\left(\alpha \approx 5^{\circ}\right)$ is lower for the highest upstream velocity, suggesting an effect of Reynolds number on the displacements. This figure also demonstrates the accuracy of the present method to measure the tip section displacements. 


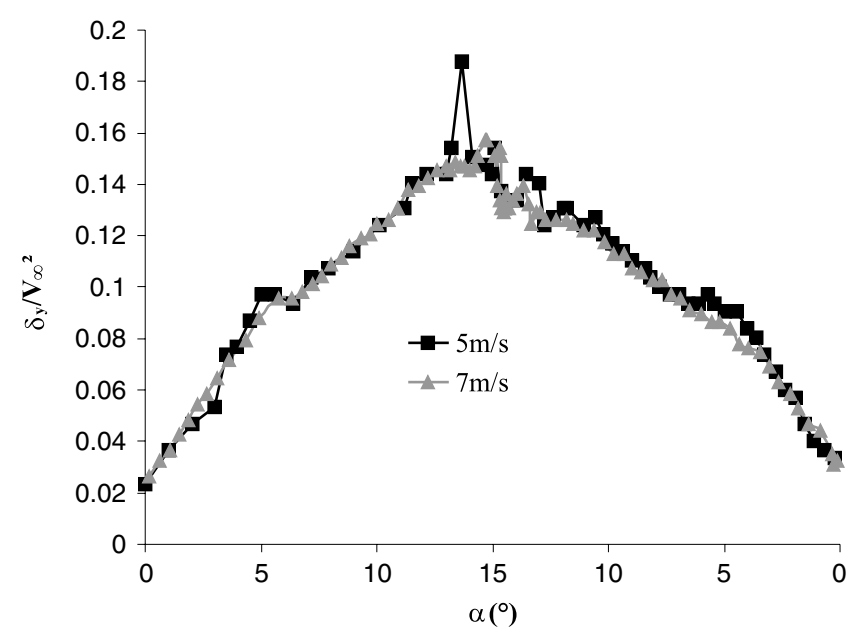

Fig. 10. Flow velocity effect: maximum displacement for 2 upstream velocities, $\dot{\alpha}^{*}=0.18$

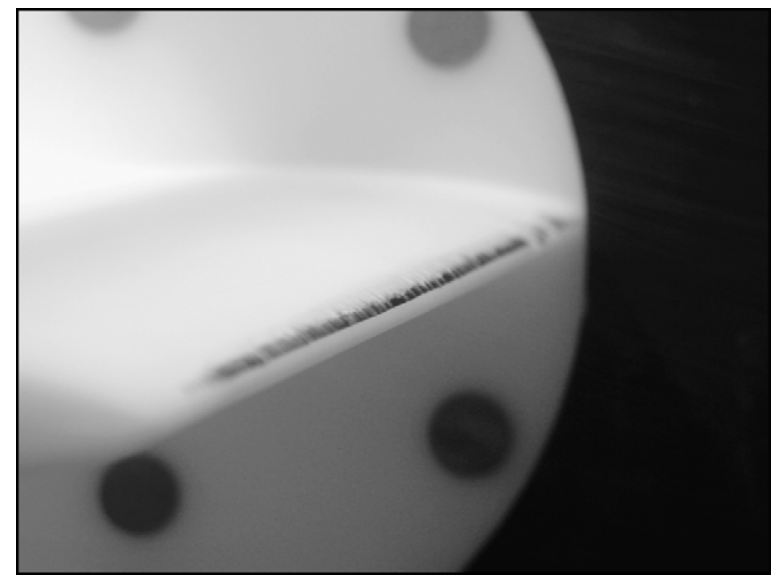

Fig. 11. Cavitation at inception at the leading edge for a fixed angle of incidence, $\alpha=8^{\circ}, \sigma=3, \operatorname{Re}=750000, V_{\infty}=5 \mathrm{~m} / \mathrm{s}$.

\subsection{Cavitation-structure interaction}

An investigation of the fluid structure interaction in the case of cavitating flows was carried out experimentally for a static angle of incidence and for the pitching motions $\dot{\alpha}^{*}=0.18$ and $\dot{\alpha}^{*}=1.89$.

\section{Static case}

Maximum displacement is measured with the same method as for non cavitating flows. A video camera is placed on the top in order to observe the cavitation development through the span (Fig. 1). The cavitation is obtained by decreasing the pressure in the tunnel test section. The cavitation inception for $\sigma=3$ is obtained at $\alpha^{+}=8^{\circ}$, as shown in Fig. 11. It is characterized by a thin band located downstream the laminar separation bubble at the leading edge of the hydrofoil.

The tip section displacement for different cavitation number was measured. Fig. 12 shows the maximum displacement versus the maximum cavity length. The development of the cavity up to $10 \%(\sigma=2.6)$ have a few effects on the displacement. A slight decrease is observed due to the inception of vapor at a higher pressure level than the minimal pressure present in subcavitating flow, which effectively reduced the hydrodynamic loading at inception (see [31]). From a cavity length of about $20 \%$, the development of cavitation increase the hydrofoil displacements. The maximum is found for $\sigma=2.05$ where the maximum displacement has increased of about $14 \%$ compared to $\sigma=7$.

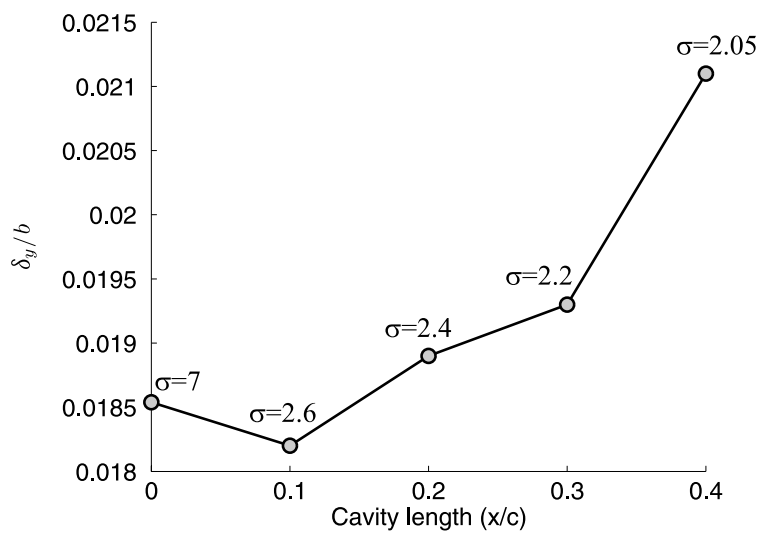

Fig. 12. Maximum displacement versus maximum cavity length along the chord, $\alpha=8^{\circ}, \operatorname{Re}=750000, V_{\infty}=5 \mathrm{~m} / \mathrm{s}$

The Fig. 14 shows the vibration spectrum for three cavitation numbers at $\alpha=8^{\circ}$. It is also observed that the hydrofoil vibrations increase globally with the cavitation development. For $\sigma=7$ (no cavitation), the first modes of vibrations are observed. For $\sigma=2.6$, the cavity length is about $10 \%$ and the excitation is clearly observed around $f=68 \mathrm{~Hz}$, corresponding to the cavity oscillation. As a consequence, the natural frequencies are excited, in particular the fundamental bending mode (mode 1). Moreover, the value is modified to $f=47.5 \mathrm{~Hz}$ (against $f=42 \mathrm{~Hz}$ for the non cavitating case). For $\sigma=2$, a pulsating cavity is observed at a main frequency of $f=27.5 \mathrm{~Hz}$ (Fig. 13). The cavity shedding is clearly shown in the spectrum, but induces also an harmonic at $f=55 \mathrm{~Hz}$ with a higher amplitude than the fundamental frequency of excitation. The response of the first bending mode and the first torsional mode are particularly increased.

The influence of the excitation frequency on the value of the two first modes are clearly shown. The other natural frequencies are submitted to the variation of the added mass due to the presence of cavitation. The Fig. 15 show an increase of the frequency of mode 4 of respectively $3 \%$ and $8 \%$ for $\sigma=2.6$ and $\sigma=2$. Indeed, the development of vapor at the hydrofoil surface decreases the fluid density on the foil surface resulting in a decrease of the added mass.

\section{Dynamic case}

The pitching motions defined for the non cavitating case are performed for $\sigma=3$, close to the cavitation number of inception for $\alpha=8^{\circ}$ (Fig. 11). Fig. 16 shows the cavitation inception for the two pitching velocities $\dot{\alpha}^{*}=0.18$ and $\dot{\alpha}^{*}=1.89$. For the low pitching velocity, the cavitation pattern at inception is close to the static case shown in Fig. 11 . However it is delayed by about $1^{\circ}$ due to the influence of the transient pitching motion, which stay significant compared to the inception rate of cavitation that form at the leading edge. A similar delay is observed during the downward motion, where the inception had been observed for $\alpha^{-}=7^{\circ}$. For the highest pitching velocity $\dot{\alpha}^{*}=1.89$, the cavitation pattern is changed (see Fig. 16(b)) and cavitation inception is delayed by about $3^{\circ}\left(\alpha^{+}=11^{\circ}\right)$. It is an attached cavity at the leading edge over approximately $10 \%$ of the chord. The delay and modification in cavitation inception can be related to the delay of the laminar to turbulent transition and laminar separation bubble at the leading edge when pitching velocity increases.

As the angle of attack increases, differences are visible in the cavitation patterns for the different pitching velocities. The lowest pitching velocity is characterized by small cavities, as show in Fig. 17. The length increases up to $0.4 c$ and have a relatively stable behavior. Small shedding of vapor structures are observed and tend to develop when the cavity length increases. For $\alpha^{+}=14^{\circ}$, a cavitation induced by vortex shedding is observed. It is characterized 

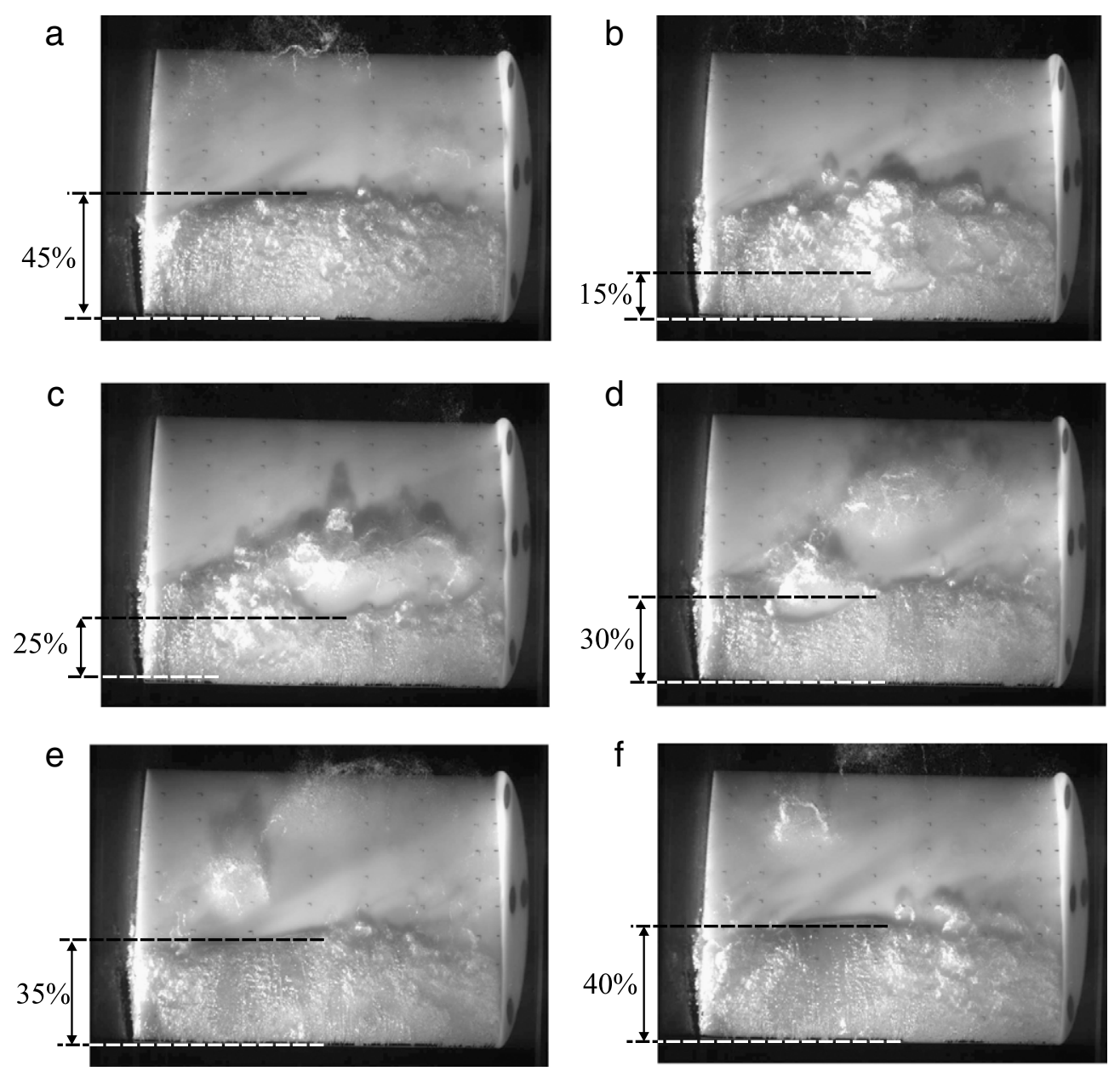

Fig. 13. Instantaneous cavity length, $\sigma=2, \alpha=8^{\circ}$, Re $=750000, V_{\infty}=5 \mathrm{~m} / \mathrm{s}$.

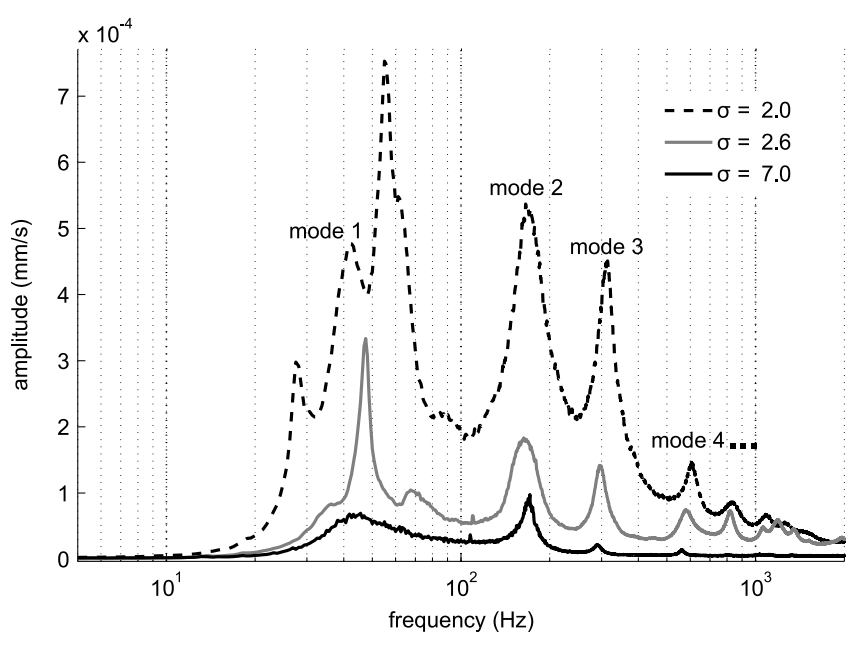

Fig. 14. Velocity spectrum of the hydrofoil for various number of cavitations, $\alpha=8^{\circ}, \operatorname{Re}=750000, V_{\infty}=5 \mathrm{~m} / \mathrm{s} . \sigma=7$ has subcavitating flow.

by spanwise streaks visible along the span, see Fig. 17(e) and (f). The same events occur when the hydrofoil goes back to $\alpha^{-}=0^{\circ}$.

For the highest pitching velocity, a relatively long sheet cavity which develops from the leading edge up to $0.6 c$ is observed (Fig. 18), together with 3D effects which appear along the span. For $\alpha^{+,-}=15^{\circ}$, the sheet cavity breaks down into a large vapor-filled structure (Fig. 18(f)) and generates a large pounding sound. This

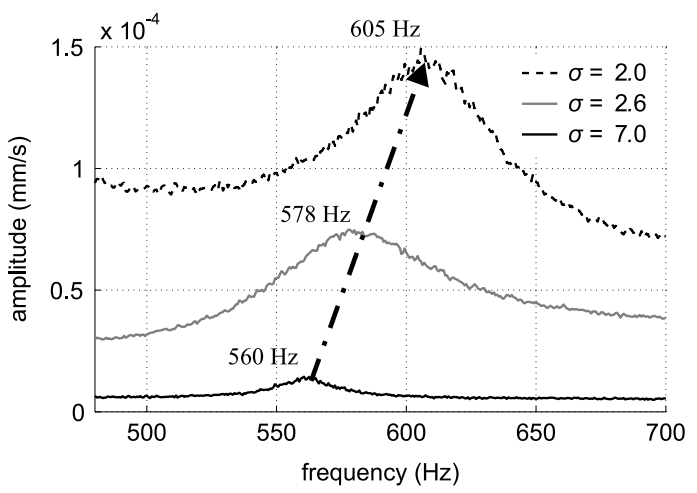

Fig. 15. Modification of the natural frequency during cavitation development, mode $4, \alpha=8^{\circ}, \operatorname{Re}=750000, V_{\infty}=5 \mathrm{~m} / \mathrm{s}$.

vapor filled structure is then convected in the wake leading edge vortex shedding pattern in Fig. 18(g). During the backward motion to $\alpha^{-}=0^{\circ}$, attached cavities appear at the leading edge and disappear quickly together with the decrease of angle of incidence (Fig. 18(h)).

The effect of cavitation on displacement during the pitching motion is shown in Fig. 19 for $\dot{\alpha}^{*}=0.18$ by comparing displacements for cavitating and non cavitating flows. The angles of incidence for which cavitation appears are framed by a doted square. Outside this zone, the two measurements are in cavitating free conditions. Displacements are quite the same at the beginning of the 


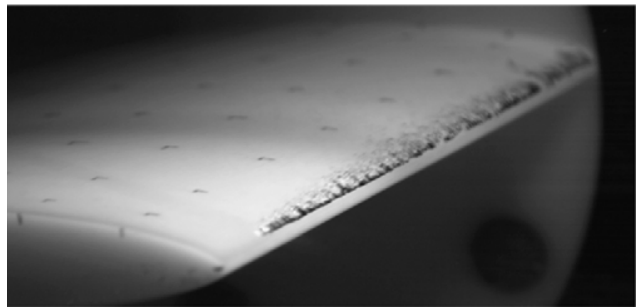

(a) $\dot{\alpha}^{*}=0.18, \alpha=9.5^{\circ}$.

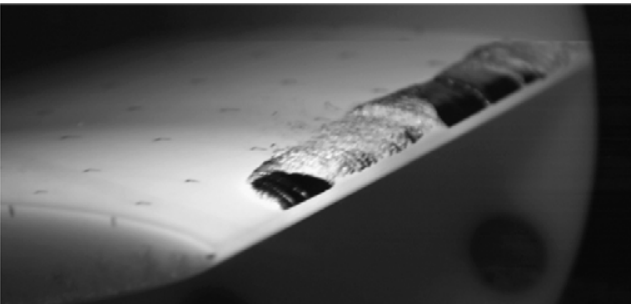

(b) $\dot{\alpha}^{*}=1.89, \alpha=11^{\circ}$.

Fig. 16. Effects of pitching velocity on cavitation inception at the leading edge, and $\sigma=3, \operatorname{Re}=750000, V_{\infty}=5 \mathrm{~m} / \mathrm{s}$.

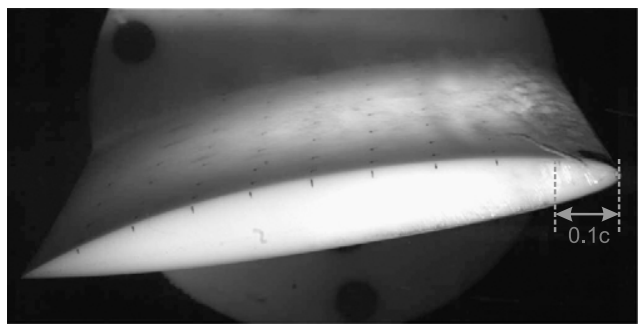

(a) $\alpha_{+}=10^{\circ}$.

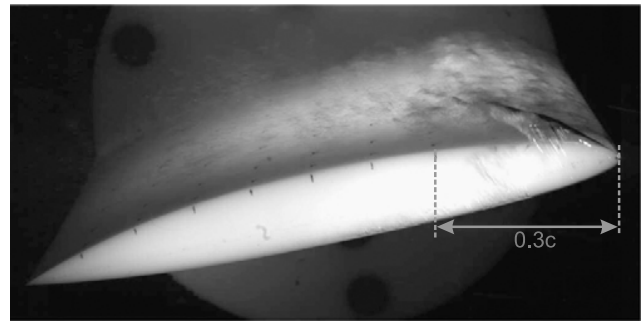

(c) $\alpha_{+}=12^{\circ}$.

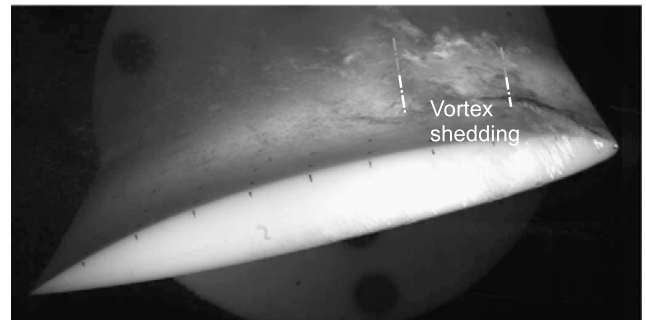

(e) $\alpha_{+}=14^{\circ}$.

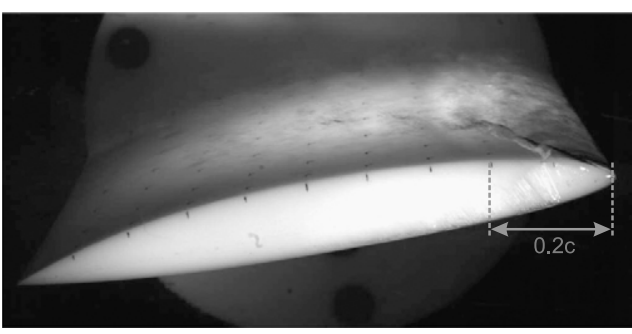

(b) $\alpha_{+}=11^{\circ}$.

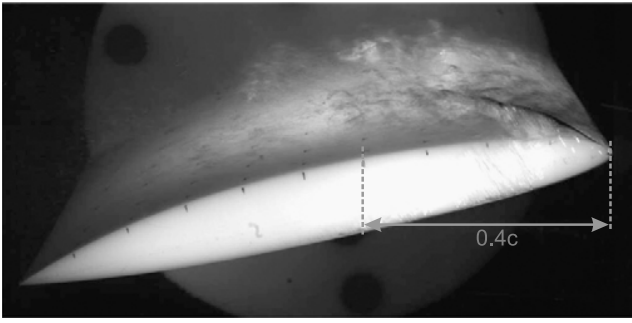

(d) $\alpha_{+}=13^{\circ}$.

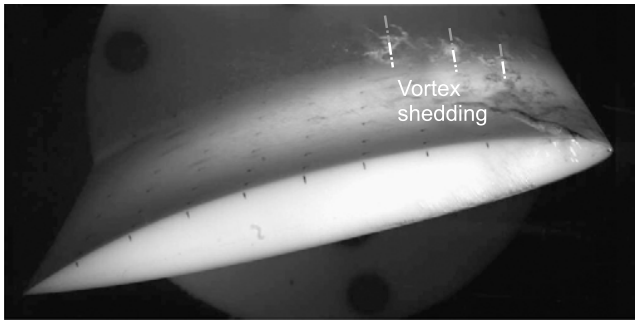

(f) $\alpha_{+,-}=15^{\circ}$.

Fig. 17. Cavitation development during the pitching motion, $\dot{\alpha}^{*}=0.18, \operatorname{Re}=750000, \sigma=3$.

motion up to the cavitation inception, except from $6^{\circ}$ to $8^{\circ}$ where a difference can be seen after boundary layer transition. Cavities increase displacement between $10^{\circ} \leq \alpha_{1} \leq 13^{\circ}$, as also observed in Fig. 21(a) at $t_{1}\left(\alpha^{+}=12^{\circ}\right)$, which show samples of the corresponding tip section displacement. This effect disappears when vortex shedding occurs and displacements fluctuates. When the flow reattaches, a slight difference is observed between the two flow conditions considered. When cavitation disappears, the same evolution is observed to the return set to $0^{\circ}$. The displacements analysis for the highest pitching velocity $\dot{\alpha}^{*}=1.89$ in Fig. 20 shows that the sheet/cloud cavitation has more influence on the structure. Displacements are higher than the case $\dot{\alpha}^{*}=0.18$ in both flow conditions, but the cavity inception at the leading edge induces a global increase of displacements. When the cavity develops on the suction side, displacements stop to increase until the implosion. A peak is then visible on Fig. 20 at $t_{2}, \alpha=15^{\circ}$, which can be observed in Fig. 21(d).

\section{Conclusion}

Experiments on a flexible hydrofoil in static and transient regimes have been carried out with original measurements techniques based on image processing and Laser Vibrometry, for non cavitating and cavitating flows.

For the non cavitating flow, the interaction between the viscous flow and the structure response was observed. The vibrations of the hydrofoil are increased by the vortex structures resulting from shedding of Laminar Separation Bubble at low angles of incidence, and leading edge vortex shedding at higher angles of incidence.

When the hydrofoil pitches, it is shown that the structural response evolves linearly with the hydrodynamic loading and that it is governed by the effect of viscosity. For small values of pitching velocity, a deplacement inflection is observed due to the laminar to turbulent transition whereas the highest pitching velocity suppresses the effect. Low frequency fluctuating displacements 


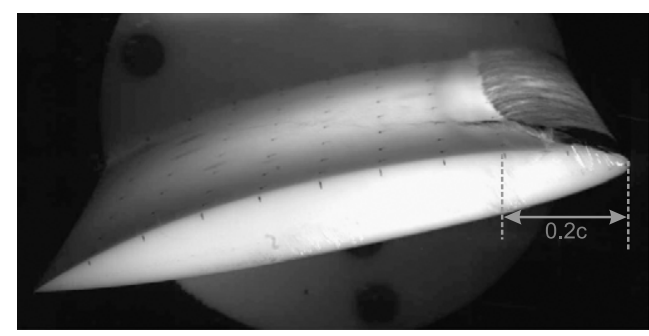

(a) $\alpha^{+}=13^{\circ}$.

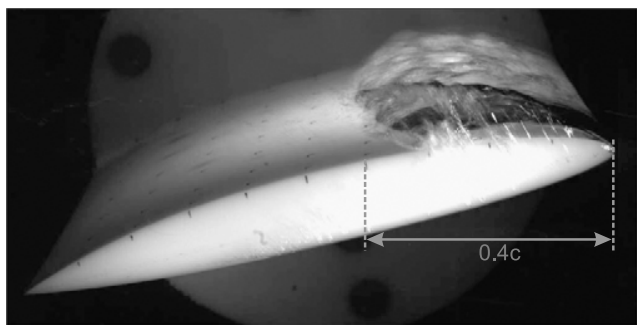

(c) $\alpha^{+}=14^{\circ}$

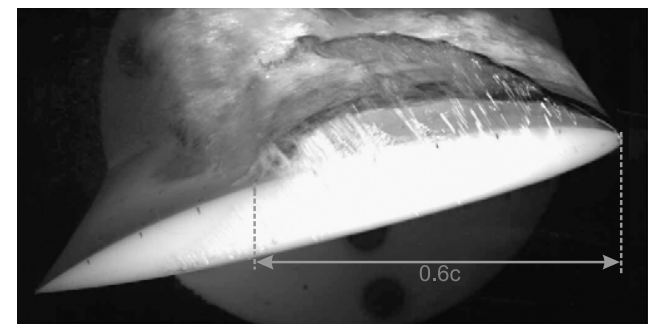

(e) $\alpha^{+,-}=15^{\circ}$.

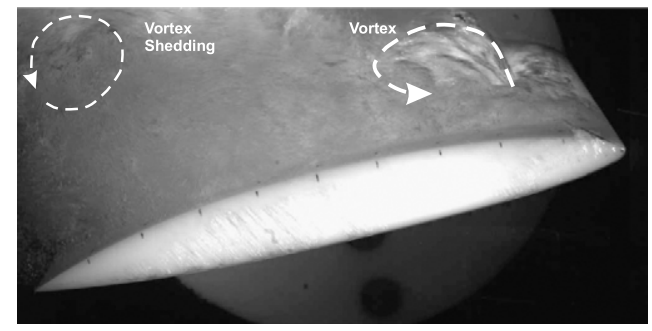

(g) $\alpha^{-}=14^{\circ}$

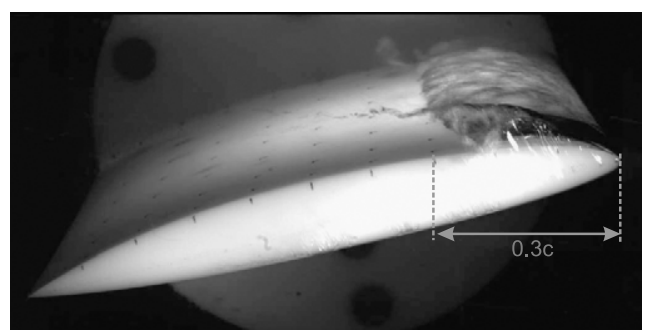

(b) $\alpha^{+}=13.5^{\circ}$.

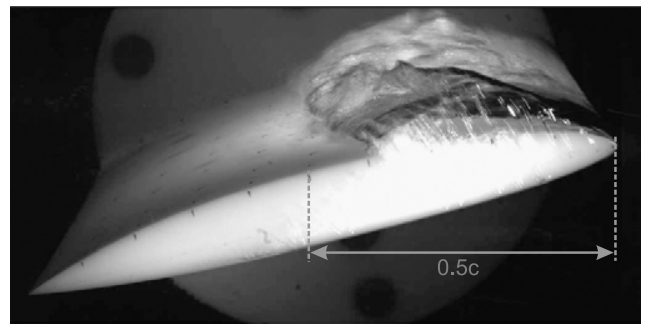

(d) $\alpha^{+}=14.5^{\circ}$.

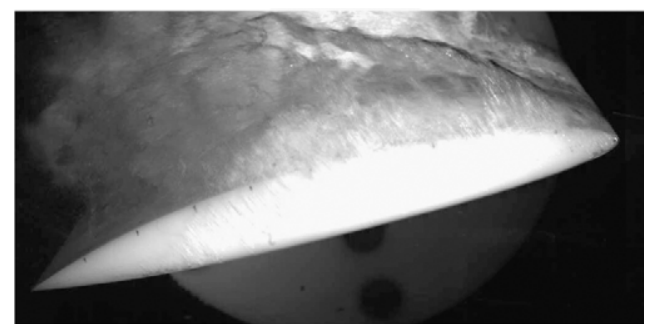

(f) $\alpha^{+,-}=15^{\circ}$.

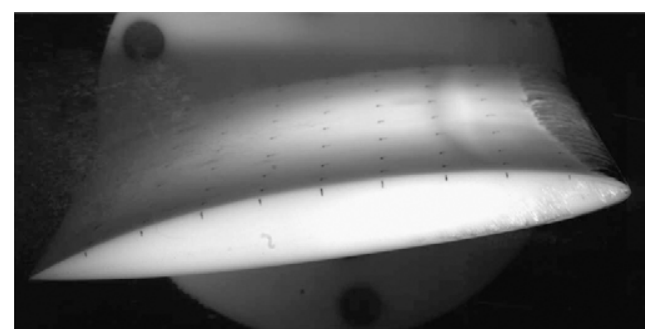

(h) $\alpha^{-}=9^{\circ}$.

Fig. 18. Cavitation development during the pitching motion, $\dot{\alpha}^{*}=1.89, \operatorname{Re}=750000, \sigma=3$.

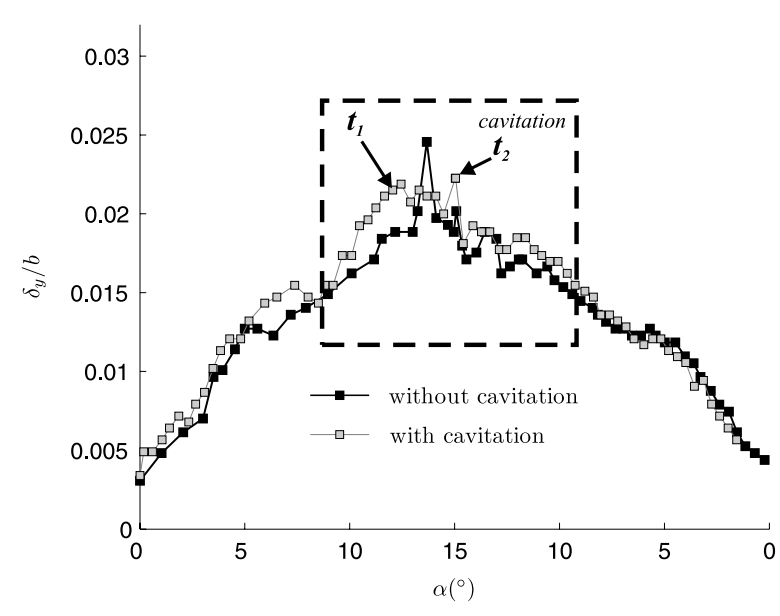

Fig. 19. Effect of cavitation on maximum displacement, $\dot{\alpha}^{*}=0.18, \operatorname{Re}=750000$, $V_{\infty}=5 \mathrm{~m} / \mathrm{s} . \sigma=3$ : cavitation; $\sigma=7$ : without cavitation.

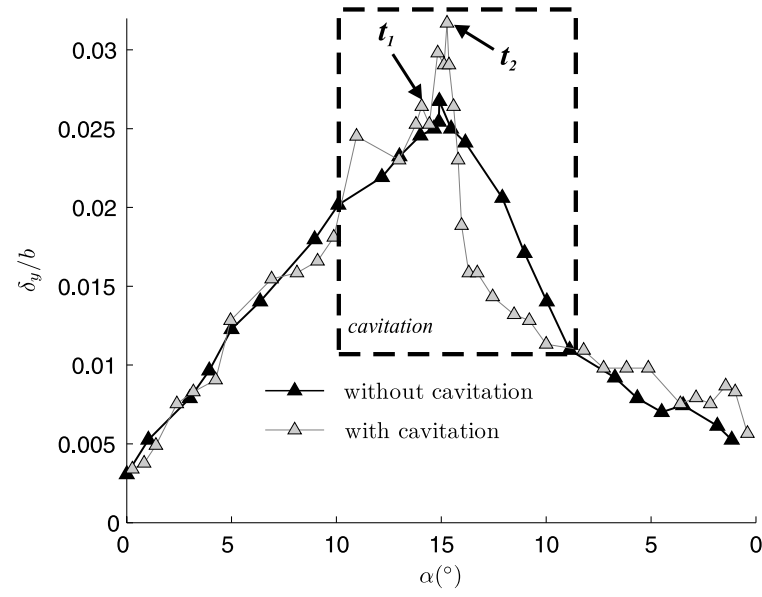

Fig. 20. Effect of cavitation on maximum displacement, $\dot{\alpha}^{*}=1.89, \operatorname{Re}=750000$, $V_{\infty}=5 \mathrm{~m} / \mathrm{s} . \sigma=3$ : cavitation; $\sigma=7$ : without cavitation. 


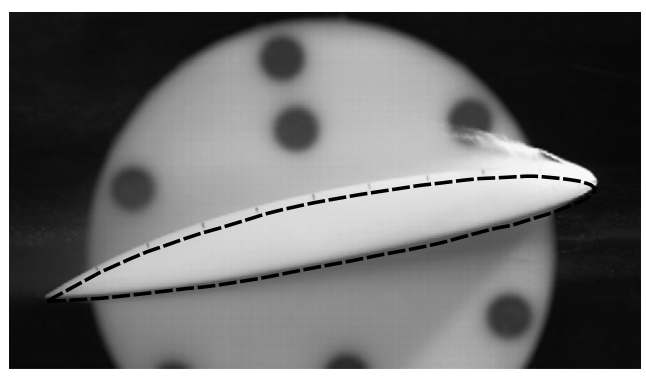

(a) $\dot{\alpha}^{*}=0.18, t_{1}, \alpha^{+}=12^{\circ}$.

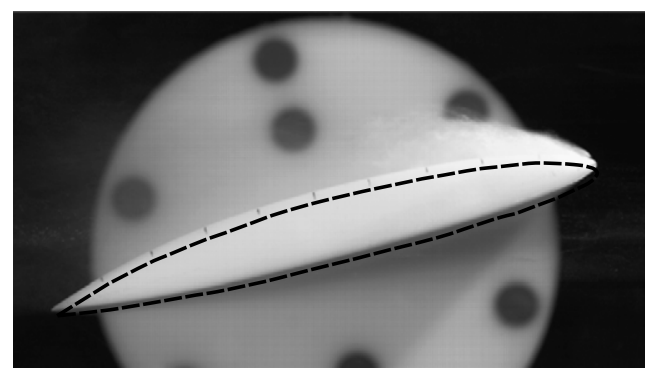

(c) $\dot{\alpha}^{*}=0.18, t_{2}, \alpha^{+,-}=15^{\circ}$.

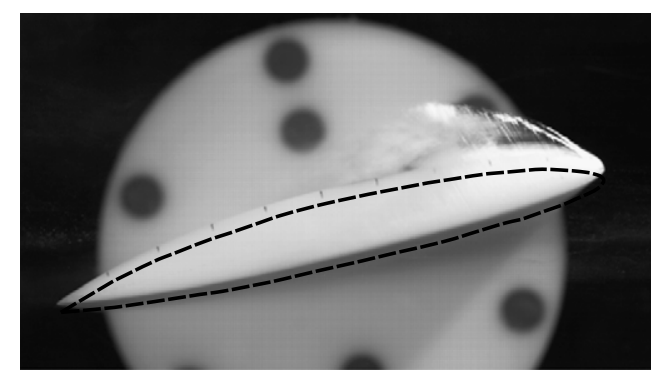

(b) $\dot{\alpha}^{*}=1.89, t_{1}, \alpha^{+}=14^{\circ}$.

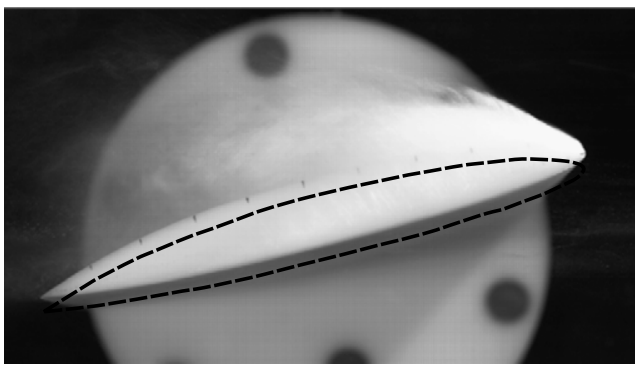

(d) $\dot{\alpha}^{*}=1.89, t_{2}, \alpha^{+,-}=15^{\circ}$.

Fig. 21. Tip section measurement for 2 pitching velocities, $\dot{\alpha}^{*}=0.18$ and $\dot{\alpha}^{*}=1.89, \operatorname{Re}=750000, V_{\infty}=5 \mathrm{~m} / \mathrm{s} . \sigma=3$.

have been observed when leading edge vortex shedding occurs during stall.

For cavitating flow, the structure vibration was measured for a fixed angle of incidence and show that cavitation highly excite the natural frequencies of the hydrofoil, in particular for pulsating cavities. The excitation of the torsional mode have probably a significant impact on cavitation structure interaction.

Measurements were also carried out in the case of cavitating flows in transient regimes, for the lowest and the highest pitching velocities. Displacements were measured and the cavitation behavior was observed at the same time by a high speed video camera. For the lowest pitching velocity small leading edge cavities are observed, then a vortex cavitation occurs at higher angles of incidences. This cavitation have few effect on the structure displacements. For the highest pitching velocity, a relatively long sheet cavity develops from the leading edge up to $0.6 \mathrm{c}$ then collapse. It has a strong impact on structure displacements. The difference of cavitation patterns between the two pitching velocities is mainly due to the pitching motion which globally suppress the viscous effects and increase the cavitation. However, the deformations can also have an influence on cavitation.

Much work has still to be done for a good understanding of flow and cavitation structure interaction applied to lifting bodies. Specific numerical coupled procedures need to be developed and can be useful in the understanding of this interaction. In this context, the experimental results exposed in this paper can be useful in the understanding of complex flow features and for the validation of these new methods.

\section{Acknowledgment}

The authors gratefully acknowledge the technical staff of IRENav for its contribution to the experimental set up.

\section{References}

[1] Y.L. Young, Fluid-structure interaction analysis of flexible composite marine propellers, J. Fluids Struct. (2008)

[2] S. Turnock, A. Wright, Directly coupled fluid structural model of a ship rudder behind a propeller, Mar. Struct. 13 (1) (2000) 53-72.
[3] R. Nicholls-Lee, S. Boyd, S. Turnock, Development of high performance composite bend-twist coupled blades for a horizontal axis tidal turbine, in: 2nd International Conference on Ocean Energy, ICOE, 15th \& 17th October, Brest, France, 2008.

[4] Y. Young, M. Motley, R. Yeung, Hydroelastic response of wind or tidal turbines, in: 28th International Conference on Ocean, Offshore and Arctic Engineering, 2009.

[5] A. Ducoin, F. Deniset, J. Astolfi, J. Sigrist, Numerical and experimental investigation of hydrodynamic characteristics of deformable hydrofoils, J. Ship Res. 53 (4) (2009) 214-226. (13).

[6] E. Jumper, S. Schreck, R. Dimnick, Lift-curve characteristics for an airfoil pitching at constant rate, J. Aircr. 24 (10) (1987) 680-687.

[7] H. Hamdani, M. Sun, Aerodynamic forces and flow structures of an airfoil in some unsteady motions at small Reynolds number, Acta Mech. 145 (1) (2000) 173-187.

[8] G.S. Triantafyllou, M.S. Triantafyllou, M. Grosenbaugh, Optimal thrust development in oscillating foils with application to fish propulsion, J. Fluids Struct. 7 (2) (1993) 205-224.

[9] T. Lee, P. Gerontakos, Investigation of flow over an oscillating airfoil, J. Fluid Mech. 512 (2004) 313-341.

[10] A. Ducoin, J. Astolfi, F. Deniset, J. Sigrist, Computational and experimental investigation of flow over a transientpitching hydrofoil, Eur. J. Mech. B/Fluids 28 (2009) 728-743.

[11] G. Kuiper, Cavitation research and ship propeller design, Appl. Sci. Res. 58 (1) (1997) 33-50.

[12] J.A. Astolfi, J.B. Leroux, P. Dorange, J.Y. Billard, F. Deniset, S. De La Fuente, An experimental investigation of cavitation inception and development on a twodimensional hydrofoil, J. Ship Res. 44 (4) (2000) 259-269.

[13] J.B. Leroux, O. Coutier-Delgosha, J.A. Astolfi, A joint experimental and numerical study of mechanisms associated to instability of partial cavitation on two-dimensional hydrofoil, Phys. Fluids 17 (2005) 052101.

[14] F. Stern, Comparison of computational and experimental unsteady cavitation on a pitching foil, J. Fluids Eng. 111 (1989) 290.

[15] D. Hart, C. Brennen, A. Acosta, Observations of cavitation on a threedimensional oscillating hydrofoil, in: ASME Cavitation and Multiphase Flow Forum, American Society of Mechanical Engineers, 1990.

[16] Y.L. Young, Time-dependent hydroelastic analysis of cavitating propulsors, J. Fluids Struct. 23 (2) (2007) 269-295.

[17] M. Engel, M. Griebel, Flow simulation on moving boundary-fitted grids and application to fluid-structure interaction problems, Internat. J. Numer. Methods Fluids 50 (2006) 437-468.

[18] M.K. Bhardwaj, A CFD/CSD interaction methodology for aircraft wings, Ph.D. Thesis, Virginia Polytechnic Institute and State University, 1997.

[19] W. Send, Coupling of fluid and structure for transport aircraft wings, in: International Forum on Aeroelasticity and Structural Dynamics, CEAS/AIAA/ICASE/NASA, Langley, Williamsburg, VA, June, 1999.

[20] M.J. Patil, D.H. Hodges, On the importance of aerodynamic and structural geometrical nonlinearities in aeroelastic behavior of high-aspect-ratio wings, J. Fluids Struct. 19 (7) (2004) 905-915. 
[21] J. Kuo, W. Vorus, Propeller blade dynamic stress, in: Proceedings, 10th Ship Technology and Research, STAR, Symposium, 1985.

[22] H.J. Lin, J.J. Lin, Nonlinear hydroelastic behavior of propellers using a finiteelement method and lifting surface theory, J. Mar. Sci. Technol. 1 (2) (1996) 114-124.

[23] E. Amromin, S. Kovinskaya, Vibration of cavitating elastic wing in a periodically perturbed flow: excitation of subharmonics, J. Fluids Struct. 14 (5) (2000) 735-751.

[24] P. Ausoni, M. Farhat, X. Escaler, E. Egusquiza, F. Avellan, Cavitation influence on von Karman vortex shedding and induced hydrofoil vibrations, J. Fluids Eng. 129 (2007) 966.

[25] M.C. Reese, Vibration and damping of hydrofoils in uniform flow, Ph.D. Thesis, Pennsylvania State University, 2010.
[26] Y.L. Young, T.J. Michael, M. Seaver, S.T. Trickey, Numerical and experimental investigations of composite marine propellers, in: Proceeding, 26th Symposium on Naval Hydrodynamics, Rome, Italy, September 17-22, 2006.

[27] M. Motley, Z. Liu, Y. Young, Utilizing fluid-structure interactions to improve energy efficiency of composite marine propellers in spatially varying wake, Compos. Struct. (2009)

[28] Y. Young, J.W. Baker, M. Motley, Reliability-based design and optimization of adaptive marine structures, Compos. Struct. (2010)

[29] J. Leroux, J. Astolfi, J. Billard, An experimental study of unsteady partial cavitation, J. Fluids Eng. Trans. ASME 126 (1) (2004) 94-101.

[30] A. Ducoin, J. André Astolfi, M. Gobert, An experimental study of boundary-layer transition induced vibrations on a hydrofoil, J. Fluids Struct. (2011)

[31] J. Carlton, Marine Propellers and Propulsion, Butterworth-Heinemann, 2007. 